\title{
Body Mass Index and Decline of Cognitive Function
}

\author{
Sujin Kim ${ }^{1}$, Yongjoo Kim², Sang Min Park ${ }^{1,3 *}$ \\ 1 Takemi Program in International Health, Harvard T. H. Chan School of Public Health, Boston, \\ Massachusetts, United States of America, 2 Department of Social and Behavioral Sciences, Harvard T. H. \\ Chan School of Public Health, Boston, Massachusetts, United States of America, 3 Department of Family \\ Medicine \& Department of Biomedical Sciences, Seoul National University College of Medicine, Seoul, South \\ Korea \\ * smpark.snuh@gmail.com
}

\section{Abstract}

\section{Background}

The association between body mass index (BMI) and cognitive function is a public health issue. This study investigated the relationship between obesity and cognitive impairment which was assessed by the Korean version of the Mini-mental state examination (K-MMSE) among mid- and old-aged people in South Korea.

\section{G open access}

Citation: Kim S, Kim Y, Park SM (2016) Body Mass Index and Decline of Cognitive Function. PLoS ONE 11(2): e0148908. doi:10.1371/journal.pone.0148908

Editor: Cheryl S. Rosenfeld, University of Missouri, UNITED STATES

Received: September 17, 2015

Accepted: January 23, 2016

Published: February 11, 2016

Copyright: $\odot 2016$ Kim et al. This is an open access article distributed under the terms of the Creative Commons Attribution License, which permits unrestricted use, distribution, and reproduction in any medium, provided the original author and source are credited.

Data Availability Statement: Data are publicly available http://survey.keis.or.kr/ENCOMAM0000N. do\#.

Funding: These authors have no support or funding to report.

Competing Interests: The authors have declared that no competing interests exist.

\section{Methods}

A cohort of 5,125 adults, age 45 or older with normal cognitive function (K-MMSE $\geq 24$ ) at baseline (2006), was derived from the Korean Longitudinal Study of Aging (KLoSA) 2006 2012. The association between baseline BMI and risk of cognitive impairment was assessed using multiple logistic regression models. We also assessed baseline BMI and change of cognitive function over the 6-year follow-up using multiple linear regressions.

\section{Results}

During the follow-up, 358 cases of severe cognitive impairment were identified. Those with baseline $\mathrm{BMI} \geq 25 \mathrm{~kg} / \mathrm{m}^{2}$ than normal-weight $\left(18.5 \leq \mathrm{BMl}<23 \mathrm{~kg} / \mathrm{m}^{2}\right)$ were marginally less likely to experience the development of severe cognitive impairment (adjusted odds ratio [aOR] = $0.73,95 \% \mathrm{Cl}=0.52$ to $1.03 ; P_{\text {trend }}=0.03$ ). This relationship was stronger among female (aOR $=0.63,95 \% \mathrm{Cl}=0.40$ to $\left.1.00 ; P_{\text {trend }}=0.01\right)$ and participants with low-normal K-MMSE score (MMSE: $24-26)$ at baseline $\left(\mathrm{aOR}=0.59,95 \% \mathrm{Cl}=0.35\right.$ to $\left.0.98 ; P_{\text {trend }}<0.01\right)$. In addition, a slower decline of cognitive function was observed in obese individuals than those with normal weight, especially among women and those with low-normal K-MMSE score at baseline.

\section{Conclusion}

In this nationally representative study, we found that obesity was associated with lower risk of cognitive decline among mid- and old-age population. 


\section{Introduction}

Overweight and obese individuals are known to be at a higher risk for dementia [1-8]. Adiposity may be directly and indirectly, by increasing other vascular risk factors, linked to cognitive impairment and dementia [9]. Nevertheless, the association between body mass index (BMI) and risk of dementia is far from clear. In late life, the detrimental consequences of being obese or overweight appear less apparent, and there are even potential protective effects. For example, individuals with high $\mathrm{BMI}\left(\mathrm{BMI} \geq 25 \mathrm{~kg} / \mathrm{m}^{2}\right)$ have been observed to have a lower risk of cognitive impairment [10-16]. Furthermore, a recent study based on a large population, almost two million, reports an inverse association between BMI and the risk of dementia in both mid- and late-life [17]. There is continuing controversy regarding the protective effects or detrimental effects of a high BMI.

The number of reported cases of dementia is rapidly increasing in Asia. By 2050, the prevalence will increase three times, and more than half of people worldwide living with dementia will live in the Asia Pacific region [18]. In particular, as Asians tend to have higher amounts of abdominal fat at lower BMIs [19], an alternative definition of overweight (BMI 23.0-24.9 kg/ $\mathrm{m}^{2}$ ) and obesity $\left(\mathrm{BMI} \geq 25 \mathrm{~kg} / \mathrm{m}^{2}\right)$ has been proposed for Asian populations [20]. For example, mortality risks significantly increased at $\mathrm{BMI} \geq 25 \mathrm{~kg} / \mathrm{m}^{2}$, rather than at $\mathrm{BMI} \geq 30 \mathrm{~kg} / \mathrm{m}^{2}[21]$. In addition, an examination of racial and ethnic differences among older adults found that the positive association of obesity with cognitive performance appeared only in Whites, and there were negative associations in Hispanics and Blacks [22]. However, while most of the existing literature has been based on western countries, the causal role of BMI as a risk factor for cognitive impairment among middle-and old-age individuals in Asian countries has rarely been investigated. Although one particular study from Asia found that obese adults experienced slower cognitive decline, it was quite small (based on fewer than 800 subjects) and short in duration of follow-up (2 years) [23]. Thus, it is unclear whether or not there is a protective association between high BMI and cognitive impairment in Asian populations.

Meanwhile, some factors may affect associations between BMI and cognitive function. For example, vascular risk factors are related to both dementia and depression [24]. Individuals with depression experience faster cognitive decline and high risk of dementia, [25-28] and also have a greater risk of vascular and neurological outcomes [29, 30], myocardial infarction and coronary heart disease [31, 32], and stroke [33, 34]. In addition, a meta-analysis showed a reciprocal positive association between obesity and depression [35] while obesity is known as a risk factor for neurobiological diseases like stroke [36, 37]. Since there might be interactions between risk factors, it could be worthwhile to examine whether the relations of obesity to cognitive function depends on other factors.

More research would be needed to better understand the relationships between obesity and cognitive impairment in Asian countries. This research investigated how BMI relates to cognitive impairment/decline in middle- and old-aged people with healthy cognitive function, using a nationally representative longitudinal sample of South Korean middle and old-aged adults.

\section{Methods}

\section{Study population and source of data}

The present study used data from the Korean Longitudinal Study of Aging (KLoSA), which is a nationally representative panel survey on middle- and old-aged populations (over 45), administered, since 2006, by the Korea Labor Institute [38]. A total of 6,171 households were sampled from 1,000 sample enumeration districts. Through the computer assisted personal interviewing procedure, data on demographics, family structure, health status, medical history, income and 
employment status for 10,254 individuals aged 45 years or older were collected in 2006 and followed-up with for every even-numbered year. Of four publicly available waves $(2006,8,10$, and 12), we used the first and fourth waves. Of the 10,254 eligible population, for whom cognitive function is measured at baseline, 7,299 individuals with normal cognitive function (the Korean version of the Mini-mental state examination, K-MMSE $>23$ ) were defined as the baseline study population [39]. 52 participants with missing values for weight and height were excluded from the first wave, and 2,122 individuals were additionally excluded from the fourth wave (326 due to death, 1,423 due to non-response, and 373 due to missing values for relevant variables). Therefore, we set our analytic sample as the remaining 5,125 respondents (Fig 1).

\section{Measurements}

The study considered a twofold dependent variable, which was measured by using the K-MMSE.: 1) onset of severe cognitive impairment; and 2) the extent of decline in cognitive functions over a six-year follow-up period. The K-MMSE included 11 items in 7 categories of cognitive functions, including orientation for time and place, registration, attention \& calculation, recall, language, and visual construction $[39,40]$. The total score of the measure ranges from 0 to 30; the higher the score, the better the cognitive function. The validity of the K-MMSE was reported elsewhere [40]. We followed the conventional classification criteria for cognitive function, categorizing K-MMSE scores as severe cognitive impairment (SCI, $\mathrm{K}-\mathrm{MMSE} \leq 17$ ), mild cognitive impairment (MCI, $18 \leq \mathrm{K}-\mathrm{MMSE} \leq 23)$, and normal cognitive function (K-MMSE $\geq 24)[39,40]$.

BMI $\left(\mathrm{kg} / \mathrm{m}^{2}\right)$ was estimated from self-reported weight and height in 2006, and was classified as underweight $(\mathrm{BMI}<18.5)$, normal $(18.5 \leq \mathrm{BMI}<23)$, overweight $(23 \leq \mathrm{BMI}<25)$, and obese $(\mathrm{BMI} \geq 25)$ according to the revised Asia-Pacific BMI criteria by the World Health Organization Western Pacific Region [20]. A validation study of the self-reported measure using a subsample $(\mathrm{N}=510)$ of KLoSA reported that there was an adequate degree of correlation between self-reported and measured BMI values (Pearson's correlation coefficient 0.837 for men, and 0.865 for women) [41].

From the first wave, other covariates were collected: age ( $45-54,55-64, \geq 65$ years), sex, marital status (married, unmarried), education (elementary, middle, high school, and $\geq$ college), equivalized household income (quartile), insurance status (Medicaid vs National Health Insurance), residence (urban, rural), cigarette smoking (yes, no), alcohol consumption (yes, no), regular physical activity (more than once a week, no), activities of daily livings ( 0 , $\geq 1$ ), depression (the Center for Epidemiologic Studies-Depression 10-item Scale $\geq 4,<4$ ), and comorbidity (at least one of hypertension, diabetes, cardiovascular disease, and/or cerebrovascular disease, 0$)$.

\section{Statistical analysis}

To investigate the association between BMI and cognitive impairment, we used two approaches in framing the outcome: 1) new-onset of SCI after 6 years' follow-up as a binary outcome (K-MMSE $\leq 17)$, and 2) cognitive decline on a continuous scale by using the change in K-MMSE scores from 2006 to 2012. As our primary approach, for the association between baseline body weight status and new-onset of SCI, we performed multiple logistic regressions for the selected analytic sample and estimated adjusted Odds Ratios (aORs). Additionally, the association between baseline body weight status and cognitive decline (change in K-MMSE scores) was assessed using multiple linear regression, and adjusted means of change were estimated. For both logistic and linear models, sets of covariates were sequentially included as follows: Model 1 (age, gender), Model 2 (marital status, health insurance, income, education, 


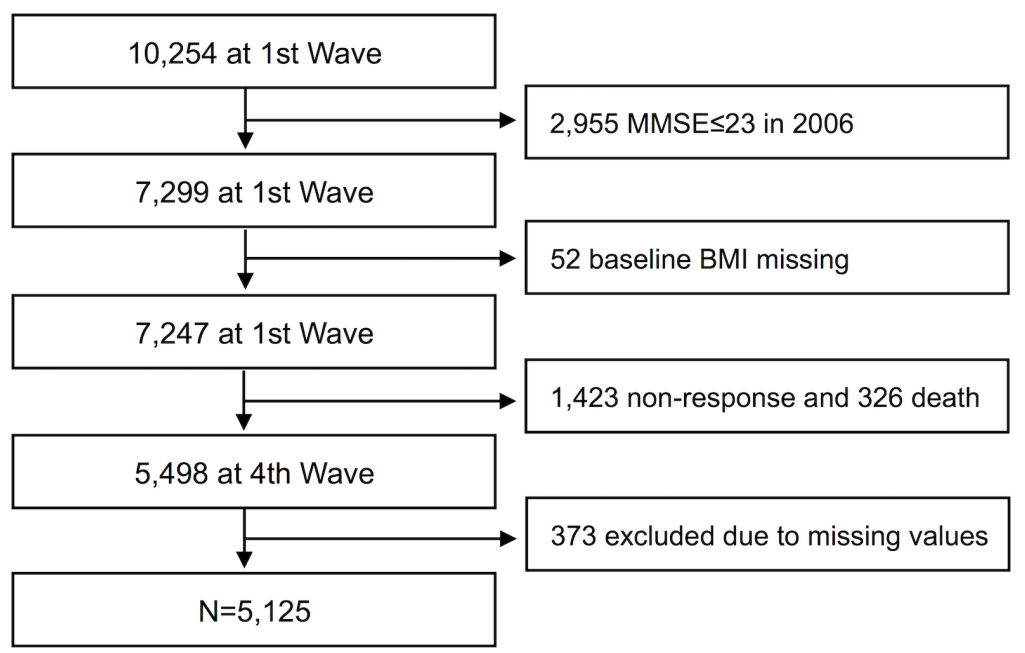

Fig 1. Schematic diagram of the study population.

doi:10.1371/journal.pone.0148908.g001

place of living, based on Model 1), Model 3 (physical activity, drinking, and smoking, based on Model 2), and Model 4 (comorbidity, activities of daily livings, depression, and baseline K-MMSE). We set Model 4 as the final model for both outcomes.

After investigating the overall impact of baseline BMI on change in cognitive function at follow-up, we performed stratified analyses by age, gender, baseline K-MMSE score (24-26, $\geq 27)$, smoking, drinking, physical activity, depression and comorbidity status in order to figure out the potentially differential impact of weight status on cognitive decline across the subgroups. We applied longitudinal sampling weight, and adjusted for complex survey design by accounting for strata and cluster. For all analyses, we used Stata ver 12.0 and set the level of significance as 0.05 (two-sided).

The study was conducted in accordance with the Ethical Principles for Medical Research Involving Human Subjects, as defined by the Helsinki Declaration. Participants were required to read and sign an agreement form before participating in the KLoSA study. For the present study, the Institutional Review Board of Seoul National University Hospital (South Korea) waved institutional review board approval because this study used only publicly available deidentified data.

\section{Results}

Table 1 presented the baseline characteristics of the study population. Among the total of 5,125 subjects, $24.6 \%$ were obese, $31.5 \%$ overweight, $41.7 \%$ normal weight, and $2.2 \%$ underweight. Across the age groups $(45-54,55-64,65+)$, the oldest group (age $\geq 65$ ) had the smallest proportions of obese (22.7\%) and overweight (30.9\%) individuals, while the group had the largest proportion of underweight (3.2\%) and normal-weight (43.2\%) individuals. Interestingly, the proportions of obese/overweight individuals were greater among those who had regular physical activity (26.9\% for obese, $32.2 \%$ for overweight) than those who did not (22.8\% for obese, $30.9 \%$ for overweight). Furthermore, the proportions of obese/overweight individuals were significantly greater among those who had chronic disease (35.5\% vs. $20.5 \%$ for obese, $32.4 \%$ vs. $31.1 \%$ for overweight) and were depressed ( $24.8 \%$ vs. $24.0 \%$ for obese, $32.6 \%$ vs. $27.4 \%$ for overweight).

Tables 2 and 3 displayed the results from the multiple logistic regression models for the association between baseline BMI and new-onset of SCI after the six year follow-up. Overall, 
Table 1. General characteristics of study population.

\begin{tabular}{|c|c|c|c|c|c|c|c|c|c|c|c|}
\hline \multirow[b]{3}{*}{ General Information } & \multirow{2}{*}{\multicolumn{2}{|c|}{$\begin{array}{c}\text { Overall } \\
\mathbf{n}=\text { Weighted } \%\end{array}$}} & \multirow{2}{*}{\multicolumn{2}{|c|}{$\frac{B M I<18.5}{n=\text { Weighted } \%}$}} & \multirow{2}{*}{\multicolumn{2}{|c|}{$\frac{18.5 \sim 23}{n=\text { Weighted } \%}$}} & \multirow{2}{*}{\multicolumn{2}{|c|}{$\begin{array}{c}\text { 23 25 } \\
\mathbf{n}=\text { Weighted } \%\end{array}$}} & \multirow{2}{*}{\multicolumn{2}{|c|}{$\frac{25+}{n=\text { Weighted } \%}$}} & \multirow[t]{3}{*}{ p-value } \\
\hline & & & & & & & & & & & \\
\hline & 5,125 & 100.0 & 116 & 2.2 & 2,169 & 41.7 & 1,599 & 31.5 & 1,241 & 24.6 & \\
\hline \multicolumn{12}{|l|}{ Age (years) } \\
\hline $45-54$ & 2,127 & 55.9 & 36 & 1.8 & 936 & 42.6 & 642 & 31.1 & 513 & 24.5 & 0.06 \\
\hline $55-64$ & 1,674 & 28.6 & 38 & 2.5 & 654 & 39.1 & 554 & 32.5 & 428 & 25.9 & \\
\hline $65+$ & 1,324 & 15.6 & 42 & 3.2 & 579 & 43.2 & 403 & 30.9 & 300 & 22.7 & \\
\hline \multicolumn{12}{|l|}{ Sex } \\
\hline Male & 2,515 & 51.3 & 56 & 1.9 & 1,034 & 39.6 & 840 & 34.3 & 585 & 24.1 & $<0.01$ \\
\hline Female & 2,610 & 48.7 & 60 & 2.5 & 1,135 & 43.9 & 759 & 28.5 & 656 & 25.2 & \\
\hline \multicolumn{12}{|l|}{ Marital status } \\
\hline Married & 4,492 & 88.5 & 91 & 2.0 & 1,919 & 42.0 & 1,397 & 31.4 & 1085 & 24.6 & 0.08 \\
\hline Unmarried & 633 & 11.5 & 25 & 3.8 & 250 & 39.8 & 202 & 31.7 & 156 & 24.7 & \\
\hline \multicolumn{12}{|l|}{ Education } \\
\hline Elementary School & 399 & 5.7 & 19 & 5.1 & 184 & 44.8 & 104 & 25.5 & 92 & 24.7 & $<0.01$ \\
\hline Middle School & 1,320 & 21.8 & 33 & 2.3 & 528 & 39.6 & 397 & 30.2 & 362 & 28.0 & \\
\hline High School & 1,011 & 19.8 & 19 & 1.9 & 405 & 39.6 & 336 & 32.5 & 251 & 26.0 & \\
\hline College+ & 2,395 & 52.7 & 45 & 2.0 & 1,052 & 43.1 & 762 & 32.2 & 536 & 22.7 & \\
\hline \multicolumn{12}{|l|}{ Household income } \\
\hline $1 Q$ & 1,305 & 22.4 & 52 & 4.0 & 552 & 41.6 & 399 & 31.4 & 302 & 23.1 & $<0.01$ \\
\hline $2 Q$ & 1,262 & 23.5 & 21 & 1.7 & 523 & 40.1 & 375 & 29.9 & 343 & 28.3 & \\
\hline $3 Q$ & 1,391 & 28.4 & 26 & 2.0 & 563 & 39.7 & 479 & 35.1 & 323 & 23.2 & \\
\hline $4 Q$ & 1,167 & 25.7 & 17 & 1.4 & 531 & 45.5 & 346 & 29.0 & 273 & 24.2 & \\
\hline \multicolumn{12}{|l|}{ Insurance } \\
\hline Medicaid & 203 & 3.9 & 9 & 3.7 & 80 & 40.9 & 56 & 27.1 & 58 & 28.4 & 0.33 \\
\hline National Health Insurance & 4,922 & 96.1 & 107 & 2.2 & 2,089 & 41.7 & 1,543 & 31.7 & 1183 & 24.5 & \\
\hline \multicolumn{12}{|l|}{ Location } \\
\hline Urban & 3,943 & 79.9 & 89 & 2.2 & 1,615 & 40.7 & 1,259 & 31.9 & 980 & 25.2 & 0.06 \\
\hline Rural & 1,182 & 20.1 & 27 & 2.1 & 554 & 45.8 & 340 & 29.6 & 261 & 22.4 & \\
\hline \multicolumn{12}{|l|}{ Cigarette smoking } \\
\hline No & 3,505 & 66.5 & 72 & 2.3 & 1,488 & 42.2 & 1,065 & 30.2 & 880 & 25.3 & 0.09 \\
\hline Yes & 1,620 & 33.5 & 44 & 2.1 & 681 & 40.7 & 534 & 34.0 & 361 & 23.2 & \\
\hline \multicolumn{12}{|l|}{ Alcohol consumption } \\
\hline No & 2,909 & 52.7 & 71 & 2.4 & 1,245 & 42.6 & 884 & 30.2 & 709 & 24.9 & 0.27 \\
\hline Yes & 2,216 & 47.3 & 45 & 2.0 & 924 & 40.7 & 715 & 32.9 & 532 & 24.3 & \\
\hline \multicolumn{12}{|l|}{ Physical Activity } \\
\hline Yes & 2,262 & 43.9 & 35 & 1.4 & 914 & 39.4 & 724 & 32.2 & 589 & 26.9 & $<0.01$ \\
\hline No & 2,863 & 56.1 & 81 & 2.8 & 1,255 & 43.5 & 875 & 30.9 & 652 & 22.8 & \\
\hline \multicolumn{12}{|l|}{ Activities of daily livings } \\
\hline 0 & 5,084 & 99.3 & 116 & 2.2 & 2,152 & 41.7 & 1,589 & 31.5 & 1227 & 24.5 & 0.31 \\
\hline $1+$ & 41 & 0.7 & 0 & 0.0 & 17 & 36.5 & 10 & 25.4 & 14 & 38.1 & \\
\hline \multicolumn{12}{|l|}{ Depression $^{a}$} \\
\hline Yes & 3,919 & 78.5 & 77 & 1.9 & 1,634 & 40.7 & 1,253 & 32.6 & 955 & 24.8 & $<0.01$ \\
\hline No & 1,206 & 21.5 & 39 & 3.3 & 535 & 45.3 & 346 & 27.4 & 286 & 24.0 & \\
\hline \multicolumn{12}{|l|}{ Comorbidity $^{\text {b }}$} \\
\hline 0 & 3,555 & 72.6 & 85 & 2.3 & 1,676 & 46.1 & 1,085 & 31.1 & 709 & 20.5 & $<0.01$ \\
\hline+1 & 1,570 & 27.4 & 31 & 2.0 & 493 & 30.1 & 514 & 32.4 & 532 & 35.5 & \\
\hline
\end{tabular}

a The Center for Epidemiologic Studies-Depression 10-item Scale $\geq 4$ (Yes), <4 (No).

${ }^{\mathrm{b}}$ Hypertension, diabetes, cardiovascular disease, or cerebrovascular diseases.

doi:10.1371/journal.pone.0148908.t001 
Table 2. Adjusted Odd Ratios of baseline body mass index for severe cognitive impairment in Korean adults $45+$ years.

\begin{tabular}{|c|c|c|c|c|c|}
\hline & \multicolumn{4}{|c|}{ Baseline body mass index } & \multirow[b]{2}{*}{$p$ for trenc } \\
\hline & $<18.5$ & $18.5 \sim 23$ & $23 \sim 25$ & $25+$ & \\
\hline All & $(n=16)$ & $(n=166)$ & $(n=110)$ & $(n=66)$ & \\
\hline Model 1 & 1.55 & 1.00 & 0.87 & 0.78 & 0.05 \\
\hline$(95 \% \mathrm{Cl})^{\mathrm{a}}$ & $(0.85-2.83)$ & & $(0.66-1.15)$ & $(0.56-1.09)$ & \\
\hline Model 2 & 1.42 & 1.00 & 0.88 & 0.76 & 0.04 \\
\hline$(95 \% \mathrm{Cl})^{\mathrm{b}}$ & $(0.76-2.64)$ & & $(0.66-1.16)$ & $(0.54-1.07)$ & \\
\hline Model 3 & 1.42 & 1.00 & 0.88 & 0.76 & 0.04 \\
\hline$(95 \% \mathrm{Cl})^{\mathrm{c}}$ & $(0.76-2.65)$ & & $(0.66-1.15)$ & $(0.54-1.06)$ & \\
\hline Model 4 & 1.44 & 1.00 & 0.87 & 0.73 & 0.03 \\
\hline$(95 \% \mathrm{Cl})^{d}$ & $(0.78-2.68)$ & & $(0.66-1.15)$ & $(0.52-1.03)$ & \\
\hline Men & $(n=7)$ & $(n=79)$ & $(n=53)$ & $(n=33)$ & \\
\hline Model 1 & 1.27 & 1.00 & 0.84 & 0.86 & 0.38 \\
\hline$(95 \% \mathrm{Cl})^{\mathrm{a}}$ & $(0.54-2.98)$ & & $(0.56-1.28)$ & $(0.53-1.39)$ & \\
\hline Model 2 & 1.18 & 1.00 & 0.86 & 0.86 & 0.43 \\
\hline$(95 \% \mathrm{Cl})^{\mathrm{b}}$ & $(0.48-2.90)$ & & $(0.56-1.31)$ & $(0.53-1.41)$ & \\
\hline Model 3 & 1.18 & 1.00 & 0.86 & 0.86 & 0.43 \\
\hline$(95 \% \mathrm{Cl})^{\mathrm{c}}$ & $(0.49-2.87)$ & & $(0.57-1.31)$ & $(0.52-1.41)$ & \\
\hline Model 4 & 1.21 & 1.00 & 0.86 & 0.83 & 0.38 \\
\hline$(95 \% \mathrm{Cl})^{d}$ & $(0.50-2.91)$ & & $(0.56-1.32)$ & $(0.49-1.41)$ & \\
\hline Women & $(n=9)$ & $(n=87)$ & $(n=57)$ & $(n=33)$ & \\
\hline Model 1 & 1.88 & 1.00 & 0.87 & 0.68 & 0.03 \\
\hline$(95 \% \mathrm{Cl})^{\mathrm{a}}$ & $(0.84-4.20)$ & & $(0.60-1.25)$ & $(0.43-1.06)$ & \\
\hline Model 2 & 1.80 & 1.00 & 0.88 & 0.66 & 0.03 \\
\hline$(95 \% \mathrm{Cl})^{\mathrm{b}}$ & $(0.79-4.10)$ & & $(0.61-1.27)$ & $(0.42-1.05)$ & \\
\hline Model 3 & 1.74 & 1.00 & 0.89 & 0.66 & 0.03 \\
\hline$(95 \% \mathrm{Cl})^{\mathrm{c}}$ & $(0.75-4.04)$ & & $(0.61-1.28)$ & $(0.42-1.05)$ & \\
\hline Model 4 & 1.77 & 1.00 & 0.87 & $0.63^{*}$ & 0.01 \\
\hline$(95 \% \mathrm{Cl})^{d}$ & $(0.77-4.05)$ & & $(0.60-1.27)$ & $(0.40-1.00)$ & \\
\hline Baseline K-MMSE:24-26 & $(n=6)$ & $(n=92)$ & $(n=67)$ & $(n=42)$ & \\
\hline Model 1 & $2.50 *$ & 1.00 & 0.71 & 0.71 & 0.02 \\
\hline$(95 \% \mathrm{Cl})^{\mathrm{a}}$ & $(1.07-5.88)$ & & $(0.46-1.09)$ & $(0.43-1.16)$ & \\
\hline Model 2 & 2.26 & 1.00 & 0.73 & 0.69 & 0.03 \\
\hline$(95 \% \mathrm{Cl})^{\mathrm{b}}$ & $(0.93-5.49)$ & & $(0.47-1.13)$ & $(0.41-1.15)$ & \\
\hline Model 3 & 2.14 & 1.00 & 0.72 & 0.69 & 0.03 \\
\hline$(95 \% \mathrm{Cl})^{\mathrm{c}}$ & $(0.87-5.29)$ & & $(0.46-1.12)$ & $(0.41-1.16)$ & \\
\hline Model 4 & 2.20 & 1.00 & 0.67 & $0.59 *$ & $<0.01$ \\
\hline$(95 \% \mathrm{Cl})^{d}$ & $(0.87-5.57)$ & & $(0.43-1.05)$ & $(0.35-0.98)$ & \\
\hline Baseline K-MMSE:27-30 & $(n=10)$ & $(n=74)$ & $(n=43)$ & $(n=24)$ & \\
\hline Model 1 & 0.96 & 1.00 & 0.97 & 0.82 & 0.38 \\
\hline$(95 \% \mathrm{Cl})^{\mathrm{a}}$ & $(0.39-2.35)$ & & $(0.69-1.37)$ & $(0.54-1.25)$ & \\
\hline Model 2 & 0.93 & 1.00 & 0.97 & 0.80 & 0.34 \\
\hline$(95 \% \mathrm{Cl})^{\mathrm{b}}$ & $(0.37-2.31)$ & & (0.69-1.38) & $(0.52-1.23)$ & \\
\hline Model 3 & 0.94 & 1.00 & 0.97 & 0.79 & 0.30 \\
\hline$(95 \% \mathrm{Cl})^{\mathrm{c}}$ & $(0.38-2.35)$ & & $(0.68-1.37)$ & $(0.52-1.21)$ & \\
\hline Model 4 & 0.95 & 1.00 & 0.98 & 0.80 & 0.36 \\
\hline
\end{tabular}


Table 2. (Continued)

\begin{tabular}{cccccc}
\hline & \multicolumn{4}{c}{ Baseline body mass index } \\
\cline { 2 - 5 } & $<18.5$ & $\mathbf{1 8 . 5} 23$ & $\mathbf{2 3} 25$ & $\mathbf{2 5 +}$ & $\boldsymbol{p}$ for trend \\
\hline$(95 \% \mathrm{Cl})^{d}$ & $(0.38-2.35)$ & & $(0.69-1.39)$ & $(0.52-1.24)$ \\
\hline
\end{tabular}

Abbreviations: K-MMSE, the Korean version of the Mini-mental state examination; $\mathrm{Cl}$, confidence interval.

$* p<.05$.

$\mathrm{n}=$ number of cases of severe cognitive impairment (K-MMSE $\leq 17$ in 2012)

Baseline body mass index was estimated from the 2006 survey.

${ }^{a}$ Adjusted for gender and age from the 2006 survey.

${ }^{\mathrm{b}}$ Based on model 1, model 2 was further adjusted for marital status, health insurance, income, educational level, and living place from the 2006 survey.

${ }^{c}$ Based on model 2, model 3 was further adjusted for physical activity, drinking, and smoking status from the 2006 survey.

${ }^{\mathrm{d}}$ Based on model 3, model 4 was further adjusted for comorbidity, baseline K-MMSE score, activities of daily livings and depression from the 2006 survey.

doi:10.1371/journal.pone.0148908.t002 
Table 3. Adjusted Odd Ratios of baseline body mass index for severe cognitive impairment by demographic and behavioral factors in Korean adults $45+$ years.

\begin{tabular}{|c|c|c|c|c|c|}
\hline & \multicolumn{4}{|c|}{ Baseline body mass index } & \multirow[b]{2}{*}{$p$ for trend } \\
\hline & $<18.5$ & $18.5 \sim 23$ & $23 \sim 25$ & $>25$ & \\
\hline Ever smoker & $(n=5)$ & $(n=60)$ & $(n=25)$ & $(n=20)$ & \\
\hline adjusted OR & 0.88 & 1.00 & $0.50 *$ & 0.62 & 0.06 \\
\hline$(95 \% \mathrm{Cl})$ & $(0.29-2.66)$ & & $(0.29-0.87)$ & $(0.33-1.16)$ & \\
\hline Never smoker & $(n=11)$ & $(n=106)$ & $(n=85)$ & $(n=46)$ & \\
\hline adjusted OR & 1.90 & 1.00 & 1.11 & 0.79 & 0.14 \\
\hline$(95 \% \mathrm{Cl})$ & $(0.94-3.86)$ & & $(0.80-1.55)$ & $(0.53-1.19)$ & \\
\hline Current drinking & $(n=2)$ & $(n=70)$ & $(n=35)$ & $(n=23)$ & \\
\hline adjusted OR & 0.46 & 1.00 & 0.65 & $0.49 *$ & 0.02 \\
\hline$(95 \% \mathrm{Cl})$ & $(0.10-2.15)$ & & $(0.41-1.03)$ & $(0.27-0.88)$ & \\
\hline No drinking & $(n=14)$ & $(n=96)$ & $(n=75)$ & $(n=43)$ & \\
\hline adjusted OR & $2.47^{*}$ & 1.00 & 1.10 & 0.98 & 0.41 \\
\hline$(95 \% \mathrm{Cl})$ & $(1.21-5.05)$ & & $(0.79-1.55)$ & $(0.65-1.49)$ & \\
\hline No physical activity & $(n=11)$ & $(n=100)$ & $(n=69)$ & $(n=33)$ & \\
\hline adjusted OR & 1.46 & 1.00 & 1.04 & 0.72 & 0.10 \\
\hline$(95 \% \mathrm{Cl})$ & $(0.68-3.13)$ & & $(0.74-1.46)$ & $(0.46-1.13)$ & \\
\hline Physical activity & $(n=5)$ & $(n=66)$ & $(n=41)$ & $(n=33)$ & \\
\hline adjusted OR & 1.45 & 1.00 & 0.68 & 0.75 & 0.22 \\
\hline$(95 \% \mathrm{Cl})$ & $(0.51-4.12)$ & & $(0.43-1.10)$ & $(0.40-1.39)$ & \\
\hline Age $\geq 65$ & $(n=10)$ & $(n=86)$ & $(n=58)$ & $(n=28)$ & \\
\hline adjusted OR & 2.02 & 1.00 & 0.94 & $0.56^{*}$ & $<0.01$ \\
\hline$(95 \% \mathrm{Cl})$ & $(0.86-4.75)$ & & $(0.64-1.38)$ & $(0.35-0.92)$ & \\
\hline Age $<65$ & $(n=6)$ & $(n=80)$ & $(n=52)$ & $(n=38)$ & \\
\hline adjusted OR & 1.14 & 1.00 & 0.81 & 0.78 & 0.19 \\
\hline$(95 \% \mathrm{Cl})$ & $(0.46-2.84)$ & & $(0.55-1.18)$ & $(0.50-1.21)$ & \\
\hline No depression & $(n=7)$ & $(n=102)$ & $(n=71)$ & $(n=52)$ & \\
\hline adjusted OR & 0.88 & 1.00 & 0.86 & 0.86 & 0.44 \\
\hline$(95 \% \mathrm{Cl})$ & $(0.39-2.01)$ & & $(0.60-1.23)$ & $(0.58-1.28)$ & \\
\hline Depression & $(n=9)$ & $(n=64)$ & $(n=39)$ & $(n=14)$ & \\
\hline adjusted OR & 2.31 & 1.00 & 0.90 & $0.42^{*}$ & $<0.01$ \\
\hline$(95 \% \mathrm{Cl})$ & $(0.90-5.96)$ & & $(0.56-1.45)$ & $(0.21-0.86)$ & \\
\hline Comorbidity: 0 & $(n=11)$ & $(n=112)$ & $(n=63)$ & $(n=32)$ & \\
\hline adjusted OR & 1.59 & 1.00 & 0.91 & 0.82 & 0.18 \\
\hline$(95 \% \mathrm{Cl})$ & $(0.78-3.24)$ & & $(0.64-1.29)$ & $(0.53-1.28)$ & \\
\hline Comorbidity: $1+$ & $(n=5)$ & $(n=54)$ & $(n=47)$ & $(n=34)$ & \\
\hline adjusted OR & 1.15 & 1.00 & 0.80 & $0.60^{*}$ & 0.04 \\
\hline$(95 \% \mathrm{Cl})$ & $(0.38-3.49)$ & & $(0.50-1.26)$ & $(0.36-1.00)$ & \\
\hline
\end{tabular}

Abbreviations: K-MMSE, the Korean version of the Mini-mental state examination; OR, Odds Ratio; Cl, confidence interval.

$* p<.05$.

$\mathrm{n}=$ number of cases of severe cognitive impairment (K-MMSE $\leq 17$ in 2012).

Baseline body mass index was estimated from the 2006 survey. Age, gender, marital status, health insurance, income, educational level, living place, physical activity, smoking status, drinking status, comorbidity, activities of daily livings, depression, and baseline K-MMSE score from the 2006 survey were adjusted for.

doi:10.1371/journal.pone.0148908.t003 
All

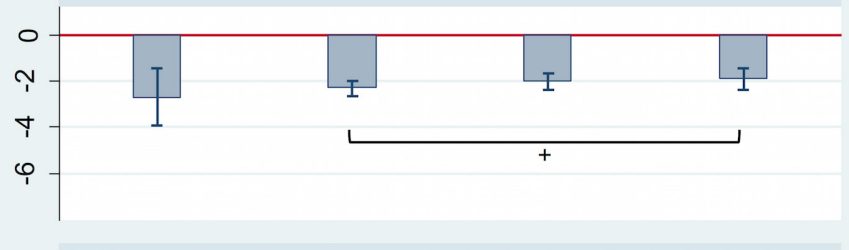

Men

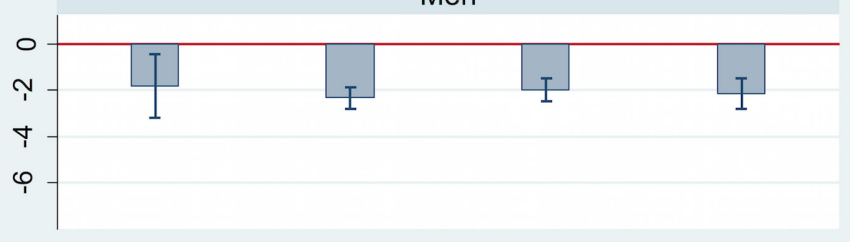

Women

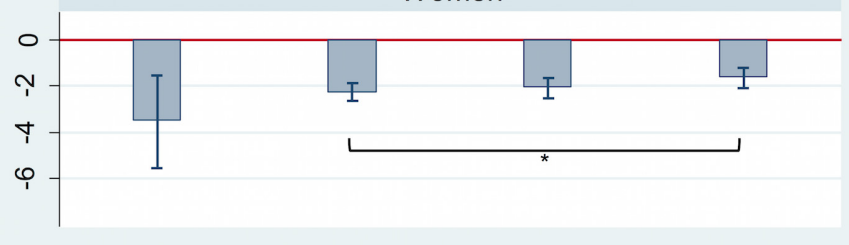

MMSE:24-26

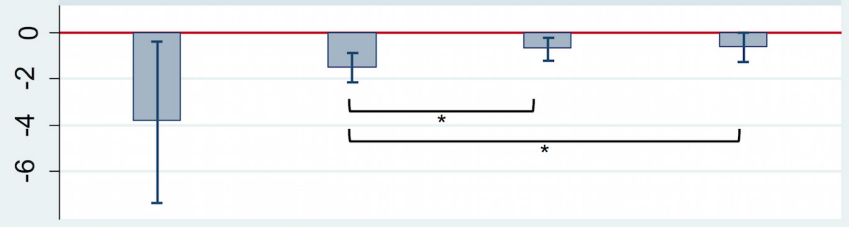

MMSE:27-30

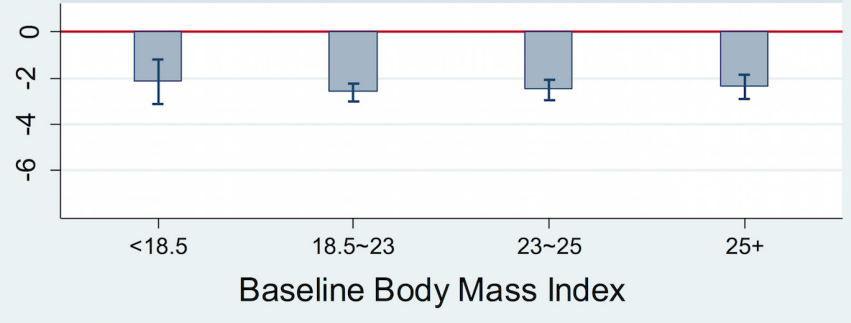

Fig 2. Adjusted mean of change of K-MMSE score across baseline body mass index in Korean adults 45+ years. Abbreviations: K-MMSE, the Korean version of the Mini-mental state examination. Reference category: body mass index $18.5-23 \mathrm{~kg} / \mathrm{m}^{2} .{ }^{*} \mathrm{p}<.05 * * \mathrm{p}<.01,{ }^{* * *} \mathrm{p}<.001$. Change of K-MMSE score was calculated by subtracting 2006 K-MMSE from 2012 K-MMSE. Baseline BMI was measured from the 2006 survey. Age, gender, marital status, health insurance, income, educational level, living place, physical activity, smoking and drinking status, comorbidity, activities of daily livings, depression, and baseline K-MMSE score from the 2006 survey were adjusted for.

doi:10.1371/journal.pone.0148908.g002

significantly smaller cognitive decline (beta for overweight $=0.80,95 \%$ CI: $0.02,1.58, \mathrm{p}=0.04$; beta for obesity $=0.88,95 \%$ CI: $0.06,1.69, \mathrm{p}=0.04)($ Fig 2$)$.

\section{Discussion}

In this nationally representative study, we provide the prospective investigation of BMI and decline of cognitive function. Our results suggest that being obese $\left(\mathrm{BMI} \geq 25 \mathrm{~kg} / \mathrm{m}^{2}\right)$, especially 
in late-life, is related to a lower risk of cognitive impairment, compared with having a normal weight $\left(18.5 \leq \mathrm{BMI}<23 \mathrm{~kg} / \mathrm{m}^{2}\right)$. This relationship became stronger when we adjusted for confounding factors, such as health behavior and health status. We also found that, although cognitive function decreased over the follow-up period, obese individuals, as compared to those with normal weight, experienced a slower decline in cognitive function. In addition, the protective association of high BMI with cognitive performance was more prominent in women and individuals with relatively low-normal K-MMSE score at baseline.

The protective association of obesity $\left(\mathrm{BMI} \geq 25 \mathrm{~kg} / \mathrm{m}^{2}\right)$ with cognitive function observed in the present study is broadly consistent with previous studies in western countries. Whereas positive association between obesity and cognitive impairment is generally expected, protective effects, rather than detrimental consequences, of a high BMI on cognitive function has often been observed, especially during old age. Older persons with high BMI scores (i.e. BMI $\geq 25$ ) at baseline have less risk of dementia $[1-8,15,42]$, and experience slower declines in cognitive function [12]. In addition, Qizilbash and colleagues [17] found inverse associations between BMI and dementia, even in mid-life, based on almost 2 million primary care patients in the UK. Another study analyzing data from the Whitehall study replicated the same results [43]. Our results showed the protective association of high BMI with cognitive impairment among adults 65 years and older. When the sample was restricted to adults $45-65$ years, the protective association was not statistically significant, and the protective relationship between high BMI and cognitive function became more evident among adults age 65 or older. Although weight loss that seemingly begins years before the onset of clinical syndrome of dementia may be related to these findings [44], it would not completely account for those associations. For instance, according to a previous study, individuals whose BMI was high (25-30) over the past four years experienced lower cognitive impairment than those with consistent normal range (20-25) of BMI [11]. Furthermore, a study with a 26-year follow-up period reported that high late-life BMI was associated with lower risk of Alzheimer's disease $(0.89,0.81-0.98)$ and marginally significant association with lower onset of dementia [7]. That is, although being underweight and losing weight may be an early marker of cognitive impairment [7, 11, 44], high BMI scores during old age tend to have consistently a protective association with cognitive function.

Meanwhile, in the present study, being underweight $(\mathrm{BMI}<18.5)$ rarely shows statistically significant associations with the development of cognitive impairment, contrary to previous findings. This is probably due to the fact that in middle- and old-age populations, being underweight has a high mortality risk [45], so cognitive impairment is possibly underestimated in the group. In addition, this study restricted the sample population to individuals with a normal range of K-MMSE scores at baseline, which in turn led the group of underweight individuals to lose more samples than other groups. Indeed, the number of underweight individuals at baseline was the smaller $(\mathrm{n}=116,2.2 \%)$ than the other weight groups.

Relationships between high BMI and cognitive impairment have been explained since adiposity is directly related to hyperinsulinemia, adipokines and cytokines, and indirectly related to vascular risk factors [9]. It is plausible that the role of BMI in dementia may change over the course of life. That is, the cut-off distinguishing underweight from normal conditions may differ in the elderly and younger populations. For example, for older people, similarly to the protective association of high BMI with cognitive function observed, the detrimental effects of high BMI on mortality are attenuated with increasing age [46], and a BMI lower than 25 correlates with a higher risk of death [47]. In addition, previous studies suggest that a BMI of less than 25 may be indicative of malnutrition for older people while nutritional and cognitive status correlates closely via the fat brain axis $[48,49]$.

The protective associations of high BMI with cognitive impairment might be related to changes in body composition. Aging is characterized by the loss of lean body mass [46], and 
higher lean body mass may be involved in reducing the risk of cognitive impairment in an older population [50]. Furthermore, high BMI may also result from increased accumulation of fat in regions other than the abdominal area. Larger leg fat mass in older individuals has been associated with improved glucose metabolism [51], which could potentially contribute to a lower risk of development of cognitive impairment. In addition, the association might be mediated by serum urate, which is positively correlated with BMI and, by acting as antioxidants in the brain, might prohibit development of neurodegenerative disease [52, 53]. Further biomarker studies are needed to clarify the relationship and mechanisms.

The present study has several limitations. First, obesity was assessed based on self-reported body weight and height in KLoSA, which can lead to biased estimates. However, the validation study reported adequate degree of correlation between self-reported and measured BMI data (Pearson's correlation: 0.84 for men, and 0.87 for women) [41]. In addition, in our modeling approach, we adjusted factors that were associated with the discrepancy in BMI measures from the validation study, including region, age, and educational level, as well as other covariates such as chronic disease histories and activities of daily livings. Second, the disagreement in BMI measures may be associated with cognitive function, which could bias the findings of the present study. However, we excluded participants with lower than normal range of K-MMSE score at baseline. Third, selection bias due to differential loss to follow-up can be an issue. Among 7,247 participants with normal cognitive function in 2006, 29\% were not included in the analysis due to death $(n=326)$, non-responses due to reasons other than death $(n=1,423)$, and missing values $(\mathrm{n}=373)$. However, the mean values of K-MMSE score at baseline were similar between the study population (mean K-MMSE score $=27.36$ for $\mathrm{N}=5,125$ ) and those who dropped out (mean score $=27.42$ for $\mathrm{N}=2,122$ ) over the follow-up. Therefore, it is possible that the potential issue of attrition of this study might not have influenced the findings of the present study. Lastly, the residuals of linear regressions for MMSE change did not appear normally distributed when the Kolmogorov Smirnov test were used. Although analysis based on log-transformation showed consistent results (i.e., There were the significantly positive associations of obesity with MMSE change among women and adults with low-normal K-MMSE score at baseline) (results not shown), the findings for MMSE changes should be interpreted more carefully.

Despite the limitations, there are several strengths of this study. Most of all, we used a nationally representative panel survey, which enables us to investigate a causal relationship between weight status to cognitive impairment/decline. To our knowledge, this is the first study that provides statistically reliable and robust evidence for an association between BMI with cognitive performance among middle- and old-age population in the region of Asia where the rate of aging-related diseases is rapidly increasing. In addition, we employed two outcome measures, both new-onset of severe cognitive impairment and cognitive decline over the follow-up, which provided consistent findings. Furthermore, we excluded individuals with mild to severe cognitive impairment (K-MMSE $\leq 23$ ) at baseline, and adjusted for a wide range of potential confounders including health-related behaviors and health status such as drinking, smoking, physical activity, activities of daily livings, depression, and comorbidities. Therefore, the findings could be robust toward potentially resolving the issue of reverse causation. Moreover, this study conducted subgroup analyses, which provide evidence for heterogeneity by a different socio-demographic characteristic. For example, we found the protective association of being obese with cognitive function is stronger among the worse-off.

In conclusion, our results suggest that being obese, especially in late-life, is related to lower risk of cognitive impairment, compared with having a normal weight. The reason for this inverse association is unclear at present. Many different issues-such as diet, frailty, and body composition change-could play a role. It is plausible that the role of BMI in dementia may 
change over the course of life. Further investigation is needed to understand the mechanism and the public health consequences of these findings.

\section{Acknowledgments}

The Korean Longitudinal Study of Aging is a public-use dataset, produced and distributed by the Korea Ministry of Employment and Labor and Korea Employment Information Service.

\section{Author Contributions}

Conceived and designed the experiments: SP. Performed the experiments: SK SP YK. Analyzed the data: SK. Wrote the paper: SK YK. Contributed to interpretation of results: SK SP YK. Contributed to revisions of the manuscript: SK SP YK.

\section{References}

1. Prickett $C$, Brennan $L$, Stolwyk R. Examining the relationship between obesity and cognitive function: $A$ systematic literature review. Obes Res Clin Pract. 2015; 9(2):93-113. Epub 2015/04/22. doi: 10.1016/j. orcp.2014.05.001 PMID: 25890426.

2. Loef $\mathrm{M}$, Walach $\mathrm{H}$. Midlife obesity and dementia: meta-analysis and adjusted forecast of dementia prevalence in the United States and China. Obesity (Silver Spring, Md). 2013; 21(1):E51-5. Epub 2013/02/ 13. doi: 10.1002/oby.20037 PMID: 23401370.

3. Dahl AK, Hassing LB, Fransson EI, Gatz M, Reynolds CA, Pedersen NL. Body mass index across midlife and cognitive change in late life. Int J Obes (Lond). 2013; 37(2):296-302. Epub 2012/03/28. doi: 10. 1038/ijo.2012.37 PMID: 22450854; PubMed Central PMCID: PMCPMC3387354.

4. Dahl AK, Hassing LB. Obesity and cognitive aging. Epidemiol Rev. 2013; 35:22-32. Epub 2012/12/22. doi: 10.1093/epirev/mxs002 PMID: 23258415.

5. Smith E, Hay P, Campbell L, Trollor JN. A review of the association between obesity and cognitive function across the lifespan: implications for novel approaches to prevention and treatment. Obesity Reviews. 2011; 12(9):740-55. doi: 10.1111/j.1467-789X.2011.00920.x PMID: 21991597

6. Xu WL, Atti AR, Gatz M, Pedersen NL, Johansson B, Fratiglioni L. Midlife overweight and obesity increase late-life dementia risk: a population-based twin study. Neurology. 2011; 76(18):1568-74 Epub 2011/05/04. doi: 10.1212/WNL.0b013e3182190d09 PMID: 21536637; PubMed Central PMCID: PMCPMC3100125.

7. Tolppanen AM, Ngandu T, Kareholt I, Laatikainen T, Rusanen M, Soininen H, et al. Midlife and late-life body mass index and late-life dementia: results from a prospective population-based cohort. J Alzheimers Dis. 2014; 38(1):201-9. Epub 2013/08/21. doi: 10.3233/jad-130698 PMID: 23948937.

8. Beydoun MA, Beydoun HA, Wang Y. Obesity and central obesity as risk factors for incident dementia and its subtypes: a systematic review and meta-analysis. Obes Rev. 2008; 9(3):204-18. Epub 2008/ 03/12. doi: 10.1111/j.1467-789X.2008.00473.x PMID: 18331422.

9. Luchsinger JA, Mayeux R. Adiposity and Alzheimererl obesity as risk factors for incident dementia an734. PMC1890025.

10. Xiang X, An R. Body weight status and onset of cognitive impairment among U.S. middle-aged and older adults. Archives of gerontology and geriatrics. 2015; 60(3):394-400. Epub 2015/03/10. doi: 10. 1016/j.archger.2015.02.008 PMID: 25747849.

11. Suemoto CK, Gilsanz P, Mayeda ER, Maria Glymour M. Body mass index and cognitive function: the potential for reverse causation. Int J Obes (Lond). 2015. Epub 2015/05/09. doi: 10.1038/ijo.2015.83 PMID: 25953125.

12. Aslan AK, Starr JM, Pattie A, Deary I. Cognitive consequences of overweight and obesity in the ninth decade of life? Age Ageing. 2015; 44(1):59-65. Epub 2014/09/25. doi: 10.1093/ageing/afu108 PMID: 25249169.

13. Anstey KJ, Cherbuin N, Budge M, Young J. Body mass index in midlife and late-life as a risk factor for dementia: a meta-analysis of prospective studies. Obes Rev. 2011; 12(5):e426-37. Epub 2011/02/26. doi: 10.1111/j.1467-789X.2010.00825.x PMID: 21348917.

14. Emmerzaal TL, Kiliaan AJ, Gustafson DR. 2003-2013: a decade of body mass index, Alzheimer's disease, and dementia. J Alzheimers Dis. 2015; 43(3):739-55. Epub 2014/08/26. doi: 10.3233/jad141086 PMID: 25147111. 
15. Hughes TF, Borenstein AR, Schofield E, Wu Y, Larson EB. Association between late-life body mass index and dementia: The Kame Project. Neurology. 2009; 72(20):1741-6. Epub 2009/05/20. doi: 10. 1212/WNL.0b013e3181a60a58 PMID: 19451529; PubMed Central PMCID: PMCPMC2683740.

16. Atti AR, Palmer K, Volpato S, Winblad B, De Ronchi D, Fratiglioni L. Late-life body mass index and dementia incidence: nine-year follow-up data from the Kungsholmen Project. J Am Geriatr Soc. 2008; 56(1):111-6. Epub 2007/11/22. doi: 10.1111/j.1532-5415.2007.01458.x PMID: 18028342.

17. Qizilbash N, Gregson J, Johnson ME, Pearce N, Douglas I, Wing K, et al. BMI and risk of dementia in two million people over two decades: a retrospective cohort study. The Lancet Diabetes \& Endocrinology. 2015; 3(6):431-6. doi: 10.1016/S2213-8587(15)00033-9

18. World Health Organization. Dementia: a public health priority. Geneva: World Health Organization, 2012.

19. Nazare J-A, Smith JD, Borel A-L, Haffner SM, Balkau B, Ross R, et al. Ethnic influences on the relations between abdominal subcutaneous and visceral adiposity, liver fat, and cardiometabolic risk profile: the International Study of Prediction of Intra-Abdominal Adiposity and Its Relationship With Cardiometabolic Risk/Intra-Abdominal Adiposity. The American Journal of Clinical Nutrition. 2012; 96(4):714-26. doi: 10.3945/ajcn.112.035758 PMID: 22932278

20. WHO Expert Consultation. Appropriate body-mass index for Asian populations and its implications for policy and intervention strategies. Lancet. 2004; 363(9403):157-63. Epub 2004/01/17. doi: 10.1016/ s0140-6736(03)15268-3 PMID: 14726171.

21. Wen CP, David Cheng TY, Tsai SP, Chan HT, Hsu HL, Hsu CC, et al. Are Asians at greater mortality risks for being overweight than Caucasians? Redefining obesity for Asians. Public Health Nutr. 2009; 12(4):497-506. Epub 2008/06/13.

22. Bryant AN, Ford K-L, Kim G. Racial/Ethnic Variations in the Relation Between Body Mass Index and Cognitive Function Among Older Adults. The American Journal of Geriatric Psychiatry. 2014; 22 (7):653-60. doi: 10.1016/j.jagp.2013.08.006 PMID: 24125815

23. Han C, Jo SA, Seo JA, Kim BG, Kim NH, Jo I, et al. Adiposity parameters and cognitive function in the elderly: application of "Jolly Fat" hypothesis to cognition. Archives of gerontology and geriatrics. 2009 49(2):e133-8. Epub 2008/12/26. PMID: 19108905.

24. Brayne $\mathrm{C}, \mathrm{Gao} \mathrm{L}$, Matthews $\mathrm{F}$. Challenges in the epidemiological investigation of the relationships between physical activity, obesity, diabetes, dementia and depression. Neurobiology of aging. 2005; 26 Suppl 1:6-10. Epub 2005/10/26. doi: 10.1016/j.neurobiolaging.2005.09.030 PMID: 16246462.

25. Yaffe K, Blackwell T, Gore R, Sands L, Reus V, Browner WS. Depressive symptoms and cognitive decline in nondemented elderly women: a prospective study. Arch Gen Psychiatry. 1999; 56(5):42530. Epub 1999/05/08. PMID: 10232297.

26. Ownby RL, Crocco E, Acevedo A, John V, Loewenstein D. Depression and risk for Alzheimer disease: systematic review, meta-analysis, and metaregression analysis. Arch Gen Psychiatry. 2006; 63 (5):530-8. Epub 2006/05/03. doi: 10.1001/archpsyc.63.5.530 PMID: 16651510; PubMed Central PMCID: PMCPmc3530614.

27. Diniz BS, Butters MA, Albert SM, Dew MA, Reynolds CF 3rd. Late-life depression and risk of vascular dementia and Alzheimer's disease: systematic review and meta-analysis of community-based cohort studies. Br J Psychiatry. 2013; 202(5):329-35. Epub 2013/05/03. doi: 10.1192/bjp.bp.112.118307 PMID: 23637108; PubMed Central PMCID: PMCPmc3640214.

28. Kohler S, van Boxtel MPJ, van Os J, Thomas AJ, O'Brien JT, Jolles J, et al. Depressive Symptoms and Cognitive Decline in Community-Dwelling Older Adults. Journal of the American Geriatrics Society. 2010; 58(5):873-9. doi: 10.1111/j.1532-5415.2010.02807.x WOS:000277414400011. PMID: 20374396

29. Schuurman AG, van den Akker M, Ensinck KT, Metsemakers JF, Knottnerus JA, Leentjens AF, et al. Increased risk of Parkinson's disease after depression: a retrospective cohort study. Neurology. 2002; 58(10):1501-4. Epub 2002/05/30. PMID: 12034786.

30. Shen CC, Tsai SJ, Perng CL, Kuo BI, Yang AC. Risk of Parkinson disease after depression: a nationwide population-based study. Neurology. 2013; 81(17):1538-44. Epub 2013/10/04. doi: 10.1212/WNL. Ob013e3182a956ad PMID: 24089392.

31. Rugulies R. Depression as a predictor for coronary heart disease: a review and meta-analysis1. American Journal of Preventive Medicine. 2002; 23(1):51-61. doi: 10.1016/S0749-3797(02)00439-7 PMID: 12093424

32. Van der Kooy K, van Hout H, Marwijk H, Marten H, Stehouwer C, Beekman A. Depression and the risk for cardiovascular diseases: systematic review and meta analysis. International Journal of Geriatric Psychiatry. 2007; 22(7):613-26. doi: 10.1002/gps.1723 WOS:000248519100001. PMID: 17236251

33. Dong J-Y, Zhang Y-H, Tong J, Qin L-Q. Depression and Risk of Stroke: A Meta-Analysis of Prospective Studies. Stroke. 2012; 43(1):32-7. doi: 10.1161/strokeaha.111.630871 PMID: 22020036 
34. Pan A, Sun Q, Okereke OI, Rexrode KM, Hu FB. Depression and risk of stroke morbidity and mortality: A meta-analysis and systematic review. JAMA. 2011; 306(11):1241-9. doi: 10.1001/jama.2011.1282 PMID: 21934057

35. Luppino FS, de Wit LM, Bouvy PF, Stijnen T, Cuijpers P, Penninx BW, et al. Overweight, obesity, and depression: A systematic review and meta-analysis of longitudinal studies. Archives of General Psychiatry. 2010; 67(3):220-9. doi: 10.1001/archgenpsychiatry.2010.2 PMID: 20194822

36. Suk S-H, Sacco RL, Boden-Albala B, Cheun JF, Pittman JG, Elkind MS, et al. Abdominal Obesity and Risk of Ischemic Stroke: The Northern Manhattan Stroke Study. Stroke. 2003; 34(7):1586-92. doi: 10 1161/01.str.0000075294.98582.2f PMID: 12775882

37. Thomas F, Bean K, Pannier B, Oppert J-M, Guize L, Benetos A. Cardiovascular Mortality in Overweight Subjects: The Key Role of Associated Risk Factors. Hypertension. 2005; 46(4):654-9. doi: 10.1161/01. hyp.0000184282.51550.00 PMID: 16157786

38. Korea Labor Institutue. User Guide for 2007 KLoSA. 2007.

39. Folstein MF, Folstein SE, McHugh PR. R. 007 KLoSA. 2007.s A. Cardiovascular Mortality in Overweight Subjects: The Key Role of Associated Risk Factors. Hypertension. 2005; 46(4):654-9.

40. Kang Y, Na DL, Hahn S. A validity study on the Korean Mini-Mental State Examination (K-MMSE) in dementia patients. Journal of the Korean Neurological Association. 1997; 15(2):300-8.

41. Yoon K, Jang SN, Chun H, Cho SI. Self-reported anthropometric information cannot vouch for the accurate assessment of obesity prevalence in populations of middle-aged and older Korean individuals. Arch Gerontol Geriatr. 2014; 59(3):584-92. doi: 10.1016/j.archger.2014.08.008 PMID: 25179443.

42. Fitzpatrick AL, Kuller LH, Lopez OL, Diehr P, O'Meara ES, Longstreth WT Jr., et al. Midlife and late-life obesity and the risk of dementia: cardiovascular health study. Arch Neurol. 2009; 66(3):336-42. Epub 2009/03/11. doi: 10.1001/archneurol.2008.582 PMID: 19273752; PubMed Central PMCID: PMCPMC3513375.

43. Kivimvimick AL, Kuller LH, Lopez OL, Diehr P, O'Meara ES, Longstreth WT Jr., et al. Midlife and latelife obesity and the risk of dementia: cardiova

44. Stewart R, Masaki K, Xue QL, Peila R, Petrovitch H, White LR, et al. A 32-year prospective study of change in body weight and incident dementia: the Honolulu-Asia Aging Study. Arch Neurol. 2005; 62 (1):55-60. Epub 2005/01/12. doi: 10.1001/archneur.62.1.55 PMID: 15642850.

45. Calle EE, Thun MJ, Petrelli JM, Rodriguez C, Heath CW. Body-Mass Index and Mortality in a Prospective Cohort of U.S. Adults. New England Journal of Medicine. 1999; 341(15):1097-105. doi: 10.1056/ NEJM199910073411501 PMID: 10511607

46. Stevens J, Cai J, Pamuk ER, Williamson DF, Thun MJ, Wood JL. The Effect of Age on the Association between Body-Mass Index and Mortality. New England Journal of Medicine. 1998; 338(1):1-7. doi: 10. 1056/NEJM199801013380101 PMID: 9414324.

47. Sergi G, Perissinotto E, Pisent C, Buja A, Maggi S, Coin A, et al. An adequate threshold for body mass index to detect underweight condition in elderly persons: the Italian Longitudinal Study on Aging (ILSA). J Gerontol A Biol Sci Med Sci. 2005; 60(7):866-71. Epub 2005/08/05. PMID: 16079209.

48. Coin A, Veronese N, De Rui M, Mosele M, Bolzetta F, Girardi A, et al. Nutritional predictors of cognitive impairment severity in demented elderly patients: The key role of BMI. J Nutr Health Aging. 2012; 16 (6):553-6. doi: 10.1007/s12603-012-0052-x PMID: 22659996

49. Douketis JD, Paradis G, Keller H, Martineau C. Canadian guidelines for body weight classification in adults: application in clinical practice to screen for overweight and obesity and to assess disease risk. CMAJ. 2005; 172(8):995-8. Epub 2005/04/13. doi: 10.1503/cmaj.045170 PMID: 15824401; PubMed Central PMCID: PMCPMC556034.

50. Nourhashash, Paradis G, Keller H, Martineau C. Canadian guidelines for body weight classification in adults: application in clinical practice to screen for overweight and obesity and to assess disease risk. CMAJ. 2005; 172(8):995-8. Epub 2005/04/13. doiciety. 2002;50(11):1796-801. PMID: 15824401

51. Snijder MB, Dekker JM, Visser M, Bouter LM, Stehouwer CDA, Yudkin JS, et al. Trunk Fat and Leg Fat Have Independent and Opposite Associations With Fasting and Postload Glucose Levels: The Hoorn Study. Diabetes Care. 2004; 27(2):372-7. doi: 10.2337/diacare.27.2.372 PMID: 14747216

52. Chen $\mathrm{H}$, Mosley $\mathrm{TH}$, Alonso A, Huang X. Plasma Urate and Parkinson's Disease in the Atherosclerosis Risk in Communities (ARIC) Study. American Journal of Epidemiology. 2009; 169(9):1064-9. doi: 10. 1093/aje/kwp033 PMID: 19299404

53. Euser SM, Hofman A, Westendorp RGJ, Breteler MMB. Serum uric acid and cognitive function and dementia2009 2009-02-01 00:00:00. 377-82 p. 